\title{
Multidisciplinary team response to a mass burn casualty event: outcomes and implications
}

\author{
Heather J Cleland, David Proud, Anneliese Spinks and Jason Wasiak
}

O n 7 February 2009, devastating bushfires in the state of Victoria caused one of Australia's worst-ever disasters. There were 173 people killed and more than 400 injured in the "Black Saturday" fires. " Thousands of dwellings and other infrastructure were destroyed. As a result of the fires, 19 patients with burn injuries were admitted to The Alfred Hospital (The Alfred), a major trauma hospital in Melbourne that houses the Victorian Adult Burns Service (VABS). VABS is the state's only adult burns service, treating 250-300 acute burn-injured inpatients each year.

Severe burn injury is an uncommon event in Western societies, and it is well recognised that burn health care resources are soon overwhelmed by relatively small numbers of patients with significant burn injuries in a mass casualty setting. ${ }^{2}$ Victoria's mass casualty burns plan defines a mass burns casualty as an event causing burns injuries that exceed or are likely to exceed the ability of the burns unit to manage them (five or more severe burn injuries is the figure used as a guide). ${ }^{3}$

Reports of bushfires in Canberra ${ }^{4}$ and San Diego ${ }^{5}$ have focused on pre-hospital planning and emergency department management rather than the overall experience of a multidisciplinary unit. In recent Australian history, the most similar event was the Ash Wednesday bushfires of 1983 in which 75 people died; ${ }^{6}$ however, the medical literature on these fires relates to psychological trauma. ${ }^{7,8}$ Mass casualty events that have been studied in terms of burn centre responses include the terrorist attacks in New York, NY ${ }^{9}$ and Washington, DC in $2001,{ }^{10}$ and Bali in 2002. ${ }^{11}$

Our study describes the characteristics of admitted patients, analyses the hospital response, and highlights the resource requirements for an adequate response to this incident. The circumstances of the injuries placed a number of these people at high risk of infective and other complications.

\section{METHODS}

\section{Data collection}

A retrospective chart review was performed on all patients who were admitted to VABS with burn injuries sustained in the February

\section{ABSTRACT}

Objectives: To describe the characteristics of patients with burn injury admitted to a major trauma hospital in Melbourne following the Black Saturday bushfires of 7 February 2009, and to provide a detailed analysis of the hospital's response to the crisis.

Design, setting and participants: A retrospective chart review of ambulance and hospital records of patients admitted to the Victorian Adult Burns Service (VABS) at The Alfred Hospital (The Alfred) following the bushfires.

Main outcome measures: Patient characteristics and outcomes: age, sex, total and full thickness body surface area burnt, type and site of burn, hospital and intensive care unit length of stay (LOS) and receipt of standard burn care practices. Estimated glomerular filtration rate, theatre time and LOS data for the bushfire cohort compared with corresponding data for historical cohorts from VABS and from a similar institution in New Zealand.

Results: Nineteen patients were admitted to VABS over the first 48 hours after the bushfires. Of these, nine patients were subsequently admitted to The Alfred's intensive care unit. Most patients (74\%) were men with a mean age of 52.7 years (SD, 12.4 years). Seventeen patients (89\%) underwent at least one surgical procedure, which resulted in 4355 minutes of theatre time for the bushfire cohort in the first week. Hospital LOS was similar for the bushfire and New Zealand cohorts. Compared with the VABS historical cohort, there was a higher incidence of abnormal renal function among the bushfire cohort patients.

Conclusions: Although relatively few patients with severe burns were admitted to VABS, significant increases in resource allocation were required to manage them in terms of additional theatre time, consumables and staffing. The experience of VABS may aid planning for future mass burns casualty events.

MJA 2011; 194: 589-593

2009 bushfires. We collected data from Ambulance Victoria records, and from The Alfred's VABS data registry and operating theatre and intensive care unit (ICU) databases. This provided information on patients' age, sex, percentage of total body surface area burned (\% TBSA), percentage of full thickness body surface area burned (\%FTSA), type and site of burn, hospital and ICU length of stay (LOS), and standard burn care practices, including fluid resuscitation therapies, number of surgical procedures and overall operating theatre time.

For each case patient, a series of historical control patients were selected to compare renal function (measured by estimated glomerular filtration rate [eGFR]) and LOS post-burn injury. The control patients were identified from the VABS database over a 12month period (April 2008 to April 2009). Each control was matched for age ( \pm 10 years), sex, ICU admission and \%TBSA burns.

For broader comparison, we obtained additional historical data on inpatient LOS/
$\%$ TBSA $^{12}$ from the National Burn Centre at Middlemore Hospital, Manukau, New Zealand, ${ }^{13}$ which serves a similar population base to that of VABS. ${ }^{14}$ The Alfred's finance department provided data on costs, and individual units and departments provided information on additional resource allocation.

\section{Data analysis}

All data were managed and analysed using Microsoft Excel (Microsoft Corporation, Redmond, Wash, USA) and OpenEpi (Open Source Epidemiologic Statistics for Public Health, version 2.3.1; http://www. openepi.com). Continuous data are presented as mean (SD) for symmetrical data, and as median (interquartile range [IQR]) or odds ratio (OR) (95\% CI) for asymmetrical data.

\section{Ethics}

Our study was approved by The Alfred's human research ethics committee. 
1 Time intervals for stages of care for patients with burn injury admitted to the Victorian Adult Burns Service (VABS) following the February 2009 bushfires

\begin{tabular}{lcccc} 
Time interval & $\begin{array}{c}\text { No. of } \\
\text { patients }\end{array}$ & $\begin{array}{c}\text { Mean } \\
\text { (hours) }\end{array}$ & SD & $\begin{array}{c}\text { Range } \\
\text { (hours) }\end{array}$ \\
\hline Receipt of documented first aid & $8(42 \%)$ & 2.17 & 2.80 & $0-8$ \\
Time to documented ambulance arrival & $14(74 \%)$ & 2.16 & 2.02 & $0.33-8$ \\
Time from injury to VABS & $19(100 \%)$ & 6.62 & 4.92 & $1.13-20$
\end{tabular}

\section{RESULTS}

\section{Transport from scene to VABS}

Nineteen patients injured in the Black Saturday bushfires were admitted to VABS. Each patient's trauma triage flow sheet and history detailing pre-hospital first aid interventions and treatments by Ambulance Victoria were analysed (Box 1)

\section{Admission to VABS}

The characteristics of patients admitted to VABS are shown in Box 2. Patients were mostly men, with a mean age of 52.7 years (SD, 12.4 years; range, $31-77$ years). Nine of the 19 patients were admitted to the ICU with burns ranging up to 50\% TBSA (mean $=18.4 \% ;$ SD, 14.5\%). The \%FTSA ranged up to $35 \%$ (mean $=11.0 \%$; SD, $12.6 \%)$. All patients received intravenous fluid resuscitation in the first 24 hours of injury (mean $=7.26 \mathrm{~mL} / \mathrm{kg} / \% \mathrm{TBSA}$; SD, 3.5 $\mathrm{mL} / \mathrm{kg} / \% \mathrm{TBSA}$; range, $3.62-16.25 \mathrm{~mL} / \mathrm{kg} /$ $\%$ TBSA). Data on urine output are not presented, as urine output was not recorded for all patients. Injuries were predominantly caused by radiant heat, with most patients suffering burns to their hands, upper and lower limbs, and head and neck region (Box 2). Other injuries included four inhalational injuries (21.1\%), two corneal abrasions (10.5\%), one foreign body injury (5.3\%), and a likely episode of severe hypoxia.

\section{Surgical response}

Seventeen patients (89\%) underwent at least one surgical procedure (mean $=2.6 ; \mathrm{SD}$, 2.04; range, 0-8), with a mean total operating time of 350.1 minutes (SD, 292.9 minutes). Thirteen patients (68\%) underwent a surgical procedure in the first 24 hours after injury, of which two occurred at other hospitals. Six patients required at least one emergency escharotomy, and eight patients required transfusion of packed red blood cells during their admission (mean = 17.5 units; SD, 13.1 units; range, 142 units). Synthetic skin substitutes were used in nine patients (47.3\%) and six patients $(32 \%)$ required the use of cadaver skin due to contaminated wound sites. Total operating theatre time and operating time by $\%$ TBSA are shown in Box 3. There was an average requirement of 16.5 minutes of operating time and 26.1 minutes of total theatre time per \% TBSA. All primary surgery was completed by Day 33 (Box 4). After the first week, there was a rapid decrease in theatre-time requirements (Box 5). Historical data from Middlemore Hospital shows a comparable theatre-time requirement of 22.8 minutes/\% TBSA burn for patients with burns $>10 \%$ TBSA, with operating time greatest in the first week and roughly halving in each subsequent week. ${ }^{12}$

\section{Complications}

Complications are summarised in Box 6. Many patients had a common history of wound contamination through contact with water in dams and cattle troughs, or with dirt and ash after injury. Given delays in transfer and difficulty ensuring adequate clinical wound surveillance, we considered infection risk to be elevated. Thus wound cultures and specimens were obtained at every dressing change and theatre visit, with all positive cultures treated with antibiotics. Repeated bronchoscopies were performed in patients with evidence of inhalation of dust and smoke.

\section{Hospital and recovery outcomes}

Of the 19 patients admitted to VABS, two died in hospital: one presented with multisystem organ failure and did not recover; the second died of respiratory failure due to fungal airway infection superimposed on severe inhalation injury.

Duration of hospital LOS ranged from 0 to 46 days (median = 22 days; IQR, 11.7529.5 days). Nine patients were admitted to the ICU, with LOS ranging from 2 to 24 days (median = 13.5 days; IQR, 6.25-16.5 days). Nine patients required mechanical ventilation (mean hours ventilated $=222$. SD, 118.9). Time to wound healing in days was reported in 13 patients (mean $=39.7$ days; SD, 13.6 days).
2 Characteristics and injuries of patients admitted to the Victorian Adult Burns Service following the February 2009 bushfires

\begin{tabular}{|c|c|}
\hline Characteristic/injury & No. of patients \\
\hline \multicolumn{2}{|l|}{ Sex } \\
\hline Male & $14(73.7 \%)$ \\
\hline Female & $5(26.3 \%)$ \\
\hline \multicolumn{2}{|l|}{ Age (years) } \\
\hline$<35$ & 1 (5.3\%) \\
\hline $36-49$ & $6(31.6 \%)$ \\
\hline $50-64$ & $8(42.1 \%)$ \\
\hline 65 & $4(21.1 \%)$ \\
\hline \multicolumn{2}{|l|}{ TBSA } \\
\hline$<10 \%$ & $5(26.3 \%)$ \\
\hline $10 \%-19 \%$ & 7 (36.8\%) \\
\hline $20 \%$ & $7(36.8 \%)$ \\
\hline \multicolumn{2}{|l|}{ FTSA } \\
\hline$<10 \%$ & 12 (63.2\%) \\
\hline $10 \%-19 \%$ & 2 (10.5\%) \\
\hline $20 \%$ & $5(26.3 \%)$ \\
\hline \multicolumn{2}{|l|}{ Burn location } \\
\hline Hands & 15 (78.9\%) \\
\hline Upper limbs & 14 (73.7\%) \\
\hline Feet & 7 (36.8\%) \\
\hline Lower limbs & $12(63.2 \%)$ \\
\hline Head/neck & $10(52.6 \%)$ \\
\hline Trunk & $6(31.5 \%)$ \\
\hline
\end{tabular}

TBSA = total body surface area burned.

FTSA = full thickness body surface area burned.

Eleven of the 17 surviving patients were discharged to a hospital-in-the-home program. The LOS in this program ranged from 7 to 29 days (mean $=17$ days; SD, 6.3 days). Three patients were discharged to another hospital or rehabilitation facility, and three were discharged home.

The extra resource allocations required in various departments are shown in Box 7 . Additional costs for hospital medical officers and consultants were estimated at A $\$ 64000$, and extra operating theatre costs (staffing and consumables) for the month of February were estimated to be A $\$ 61500$.

Inpatient LOS/\%TBSA was similar for both the VABS bushfire and the Middlemore Hospital cohorts (1.0 days and 1.1 days, respectively).

Median hospital LOS was greater for the VABS bushfire cohort than for the VABS historical cohort (21 days [IQR, 12-27 days] v 13 days [IQR, 4-28.5 days]). The VABS 
bushfire cohort had a higher incidence of acute renal impairment (defined by abnormal eGFR levels) compared with those of the VABS historical cohort (68\% v 55\%; OR, 1.75 [95\% CI, 0.55-5.59]).

\section{DISCUSSION}

Burns > 20\% TBSA are considered "severe" in the setting of a mass casualty incident, and seven patients admitted to VABS as a result of the bushfires had what is defined as a severe burn injury. To put this in context, an average of 35 patients a year in the state of Victoria received burns of $>20 \%$ TBSA in the years 20002006. ${ }^{15}$

The requirement for ICU beds when treating burn patients is frequently the limiting factor in the acute response to mass casualty incidents, ${ }^{16}$ as patients with airway injury or extensive burns necessitating large doses of opiates for analgesia require intubation and ventilation. The acute creation of 10 additional ICU ventilator beds at The Alfred has been documented elsewhere. ${ }^{1}$

Disaster plans focus on the triage and distribution of casualties to hospitals. While it has been increasingly recognised in recent years that burns patients require large amounts of resources human, infrastructural and consumable - what this actually means in terms of capacity is difficult to quantify. The delayed demands on such resources are increasingly difficult to measure as time from the incident elapses, yet burns patients have notoriously prolonged and complex hospital stays, and require lengthy rehabilitation and secondary surgery.

In terms of calculating what the theatre requirements of a particular burn patient are likely to be, for the group with burns between 10\% and $50 \%$ TBSA, 21 minutes of theatre time per \% TBSA burn for the whole inpatient stay would seem to provide a reasonable estimate, based on experiences at Middlemore Hospital and VABS. This estimate can be used in the event of a mass burn casualty event to determine the ability of a hospital to
3 Theatre usage time following the Victorian bushfires, by percentage of total burn surface area (\%TBSA), February-March 2009

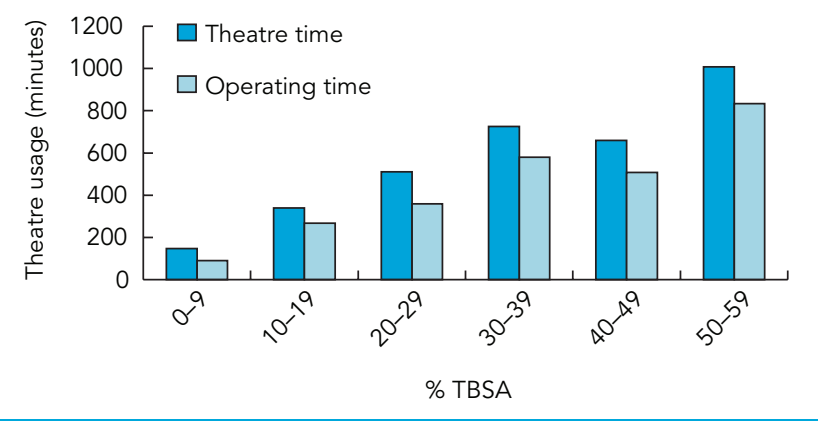

4 Daily theatre usage following the Victorian bushfires, February-March 2009

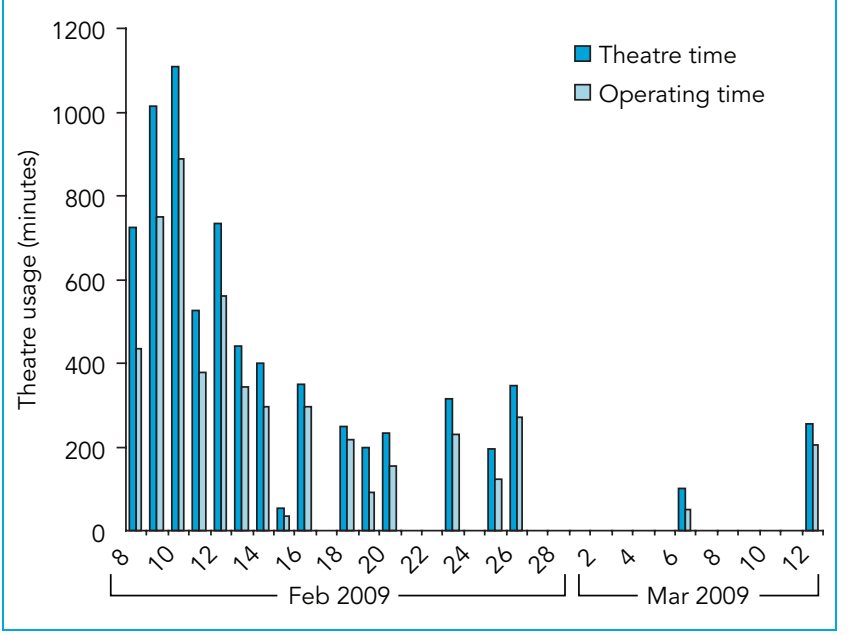

5 Total weekly theatre usage following the Victorian bushfires, February-March 2009

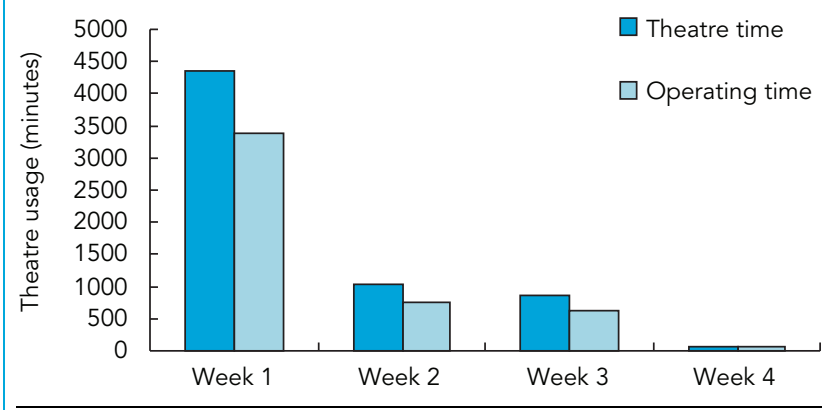

Week 1 = 8-14 Feb 2009. Week 2 = 15-21 Feb 2009 . Week 3 = 22-28 Feb 2009. Week 4 = 1-7 Mar 2009 .

estimated that for the state of South Australia, five adult patients with massive burns would consume all normal burns unit resources for at least 3 months, after requiring a minimum of 5 hours each of surgery per week for the first 3 weeks. ${ }^{17}$ A patient with $>50 \%$ TBSA burns has insufficient donor sites to provide early definitive wound closure, and is prone to a multitude of complications, making for a highly idiosyncratic clinical course. Add to this the fact that these are relatively uncommon injuries (most Australian burns units would admit around three to four such patients a year, some of whom might be treated palliatively), and it becomes very difficult to produce meaningful average data. The presence of people with burns $>50 \%$ TBSA in a mass casualty cohort increases both acute and longer-term resource requirements exponentially, as well as greatly extending the length of time of increased demands, and would significantly complicate decision making with respect to resource allocation. The recently established Australia and New Zealand Bi-National Burns Registry will provide comprehensive data on these uncommon injuries and aid in predicting resource requirements.

The ability to provide early total burn wound excision is crucial in minimising complications in burns patients ${ }^{18}$ — especially in those with severe burns (>20\% TBSA) who are at risk of developing systemic complications $^{19}$ — which consume extra resources over increasing time periods in an already pressured environment. A hospital responding to a mass burn casualty event must, as a priority, ensure that theatres and surgeons are acutely available to enable early excision of severe burns. This strategy will

provide appropriate care for the cohort of patients with 10\%-50\% TBSA burns. Theatre demands are maximal in the first week and decrease rapidly thereafter, at least halving in each subsequent week.

However, attempts to predict the requirements of patients with massive burns are far more problematic and uncertain. It has been minimise ongoing demands for resources and ensure best outcomes for patients. If excision is delayed, complications increase, hospital stays drag out, and the drain on resources limits the institution's ability to return to business as usual quickly.

In the aftermath of the 2009 bushfires, VABS was able to continue to admit and 


$\begin{aligned} & \text { Post-burn injury complications in } \\
& \text { patients admitted to the Victorian } \\
& \text { Adult Burns Service }\end{aligned}$
\begin{tabular}{ll} 
Complication & No. of patients \\
\hline $\begin{array}{l}\text { Infective } \\
\text { Blood stream infection }\end{array}$ & $2(10.5 \%)$ \\
$\begin{array}{l}\text { External auricular abscess } \\
\text { Urine }\end{array}$ & $1(5.3 \%)$ \\
$\begin{array}{l}\text { Chest infection } \\
3 \text { organisms }\end{array}$ & $1(5.3 \%)$ \\
2 organisms & $2(10.5 \%)$ \\
$\begin{array}{l}\text { 1 organism } \\
\text { Positive wound culture } \\
\text { donor site }\end{array}$ & $2(10.5 \%)$ \\
$\begin{array}{l}\text { Non-infective } \\
\text { Perioperative respiratory } \\
\text { arrest }\end{array}$ & $1(5.3 \%)$ \\
$\begin{array}{l}\text { Missed subcutaneous } \\
\text { foreign body }\end{array}$ & $1(5.3 \%)$ \\
$\begin{array}{l}\text { Non-healing wound } \\
\text { requiring split skin graft }\end{array}$ & $1(5.3 \%)$ \\
$\begin{array}{l}\text { Failed grafting requiring } \\
\text { regrafting }\end{array}$ & $1(5.3 \%)$ \\
$\begin{array}{l}\text { Retained staples requiring } \\
\text { anaesthetic for removal }\end{array}$ & $2(10.5 \%)$ \\
\end{tabular}

treat all patients who met the criteria for transfer to a specialist burns unit according to the Australia and New Zealand Burn Association guidelines. However, in a true mass casualty situation, patients with burns $<20 \%$ TBSA may well require admission to a non-specialist facility in order to allow the specialist burns referral centre to continue to manage more severe and complicated burns injuries.

The amount of fluid resuscitation in the VABS bushfire cohort is comparable to results previously obtained at the same institution. ${ }^{20}$ The mean amount of fluid received exceeded that calculated by the Parkland formula and reflects the recent trend towards "fluid creep" in burns resuscitation. ${ }^{21}$ However, many of the patients were dehydrated on presentation through a combination of burns injury, high ambient temperatures $\left(>46^{\circ} \mathrm{C}\right)$ and delayed presentation to hospital. The increased rate of renal impairment compared with the matched cohort may reflect this. The infectious complications in patients with histories of exposure to contaminated water are in keeping with recent literature. ${ }^{22}$

It is important to recognise that victims of a mass casualty event have needs beyond medical treatment. Many of our patients had lost family members, homes, livestock and their livelihoods. They had significant loss and grief issues. In addition to the provision of medical treatment, The Alfred dealt with media issues, provided psychological support, and responded to the social needs of these patients and their families as part of the statewide response to this unprecedented catastrophe.

There were relatively few patients with severe burns admitted as a result of the bushfires when compared with the number of lives lost. This may reflect the extreme conditions created by the firestorms experienced on 7 February 2009 and that most people trapped by the fires perished. Nonetheless, significant increases in resource allocation were required to manage the survivors with burn injuries. Analysis of this resource allocation can assist in planning for future mass burn casualty incidents.

\section{ACKNOWLEDGEMENTS}

We thank Richard Wong She, Department of Plastic, Reconstructive and Burn Surgery, Middlemore Hospital, Auckland, New Zealand, for providing us with additional patient data. In addition, many staff members in many departments throughout The Alfred, as well as volunteers, contributed an extraordinary amount of unpaid time and effort to ensure that the Burns Service and the rest of the hospital continued to function smoothly and provide a high standard of care to all patients and their families.

\section{COMPETING INTERESTS}

None identified.

\section{AUTHOR DETAILS}

Heather J Cleland, MB BS, FRACS, Director ${ }^{1}$

David Proud, MB BS, Surgical Trainee ${ }^{1}$ Anneliese Spinks, PhD, Research Fellow 2,3 Jason Wasiak, MPH, Research Fellow ${ }^{1}$

1 Victorian Adult Burns Service, The Alfred Hospital, Melbourne, VIC.

2 CSIRO Ecosystem Sciences, Brisbane, QLD.

3 School of Medicine, Griffith University,

Meadowbrook, QLD.

Correspondence: J.Wasiak@alfred.org.au

\section{REFERENCES}

1 Cameron PA, Mitra B, Fitzgerald M, et al. Black Saturday: the immediate impact of the February 2009 bushfires in Victoria, Australia. Med $J$ Aust 2009; 191: 11-16.

2 Cassuto J, Tarnow P. The discotheque fire in Gothenburg 1998. A tragedy among teenagers. Burns 2003; 29: 405-416.

3 Victorian Government Emergency Management Branch. Victorian mass casualty burns plan. Melbourne: Department of Human Services, 2006. http://www.dhs.vic.gov.au/_data/ assets/pdf_file/0005/69791/burns_subplan_ 2006.pdf (accessed Jan 2011).

4 Richardson DB, Kumar S. Emergency response to the Canberra bushfires. Med J Aust 2004; 181: 40-42.

5 Vilke GM, Smith AM, Stepanski BM, et al. Impact of the San Diego county firestorm on

7 Extra staff* allocated to the Victorian Adult Burns Service in the aftermath of the February 2009 bushfires

\begin{tabular}{llcl} 
Department & Level & Extra staff & Comments \\
\hline Acute Pain Service & Pain nurse & 1 & $\begin{array}{l}\text { Immediate acute doubling } \\
\text { of workload }\end{array}$ \\
& Pain consultant & 1 & Plus 100 hours of overtime \\
Infectious Diseases & Registrar & 0.25 & for laboratory staff \\
& Consultant & 0.25 & \\
Junior Medical Staff & Resident & 1 & An extra 296 nursing hours \\
Nursing & Clinical nurse specialist & 2 & allocated over 6 weeks \\
Nutrition & (ward) & & Initial tripling of workload \\
Occupational therapy & Occupational therapist & 1 & \\
& Allied health assistant & 0.5 & \\
Physiotherapy & Physiotherapist & 1 & \\
Psychiatry & Allied health assistant & 0.5 & \\
& Psychiatry nurse & 1 & Provided staff support \\
& Psychiatry registrar & 1 & service in addition to patient \\
care
\end{tabular}

* Full-time equivalent. 
emergency medical services. Prehosp Disaster Med 2006; 21: 353-358.

6 Australian Government Bureau of Meteorology. Climate education. Ash Wednesday, Feb 1983. http://www.bom.gov.au/lam/climate/levelthree/c20thc/fire5.htm (accessed Jan 2011).

7 McFarlane AC, Raphael B. Ash Wednesday: the effects of a fire. Aust NZ J Psychiatry 1984; 18 : 341-351.

8 Valent P. The Ash Wednesday bushfires in Victoria. Med J Aust 1984; 141: 291-300.

9 Yurt RW, Bessey PQ, Bauer GJ, et al. A regional burn center's response to a disaster: September 11, 2001, and the days beyond. J Burn Care Rehabil 2005; 26: 117-124.

10 Jordan $\mathrm{MH}$, Hollowed KA, Turner DG, et al. The Pentagon attack of September 11, 2001: a burn center's experience. J Burn Care Rehabil 2005; 26: 109-116.
11 Palmer D, Stephens D, Fisher DA, Spain B, et al. The Bali bombing: the Royal Darwin Hospital response. Med J Aust 2003; 179: 358-361.

12 Phua YS, Miller JD, Wong She RB. Total care requirements of burn patients: implications for a disaster management plan. J Burn Care Res 2010; 31: 935-941.

13 HealthPoint. Auckland Regional Burn Service/ National Burn Service. http://www.healthpoint.co.nz/default,28348.sm?location $=3727$ (accessed Jan 2011).

14 The World Bank. Data. New Zealand. http:// data.worldbank.org/country/new-zealand (accessed Jan 2011).

15 Wasiak J, Spinks A, Ashby K, et al. The epidemiology of burn injuries in an Australian setting, 2000-2006. Burns 2009; 35: 1124-1132.

16 Barillo DJ, Wolf S. Planning for burn disasters: lessons learned from one hundred years of history. J Burn Care Res 2006; 27: 622-634.
17 Greenwood JE, Mackie IP. Factors for consideration in developing a plan to cope with mass burn casualties. ANZ J Surg 2009; 79: 581-583

18 Mosier MJ, Gibran NS. Surgical excision of the burn wound. Clin Plast Surg 2009; 36: 617-25.

19 Chrysopoulo MT. Acute renal dysfunction in severely burned adults. J Trauma 1999; 46: 141 144.

20 Mitra B, Fitzgerald M, Cameron P, Cleland H. Fluid resuscitation in major burns. ANZ J Surg 2006; 76: 35-38.

21 Tricklebank S. Modern trends in fluid therapy for burns. Burns 2009; 35: 757-767.

22 Ribeiro NF, Heath $\mathrm{CH}$, Kierath J, et al. Burn wounds infected by contaminated water: case reports, review of the literature and recommendations for treatment. Burns 2010; 36: 9-22.

Provenance: Not commissioned; externally peer reviewed.

(Received 21 Sep 2010, accepted 17 Feb 2011) 